\title{
III. Ueber einige Anwendungsweisen des faradischen Stromes in der Gynäkologie.2)
}

\author{
Von Dr. P. Bröse in Berlin.
}

Die methodische Anwendung der Elektricität in der Gynäkologie ist eine Errungenschaft des letzten Decenniums. Es ist das grosse Verdienst A postoli's, die Aufmerlssamkeit der Gynäkologen auf dieses Gebiet der Therapie gelenlkt und neue und sichere Methoden der Elektrotherapie geschaffen zu haben. Es ist auffallend, dass bei uns in Deutschland die Gynäkologen sich bis vor ganz kurzer Zeit so gut wie gar nicht um die Elektrotherapie bekümmert haben, trotz der allgemeinen Anwendung, welche die Elektricität in der gesammten übrigen Medicin gefunden. Giebt es doch kaum ein inneres Organ, welches der Application der Elektricităt so zugänglich ist, wie der Uterus und die iibrigen Organe des weiblichen Beckens. E $r^{b^{3}}$ ) spricht in seinem Handbuch der Elektrotherapie niber die Vernachlässigung dieser Heilmethode von Seiten der Specialisten der Gynälologie seine Verwunderung aus und sagt: „Ganz besonders mïsste doch $\mathbf{z u}$ der Verwerthung der mächtigen Heilwirkungen des elektrischen Stromes der Umstand auffordern, dass der Uterus ein musculöses, sehr gefässreiches und mit zahlreichen Nervenverbindungen ausgestattetes Organ ist; den vasomotorischen und katalytischen, den erregenden und beruhigenden Wirkungen des elektrischen Stromes scheint sich hier denn doch ein weites und dankbares Feld zu eröffnen." Forscht man nach den Gründen dieser Vernachlässigung der Elektrotherapie von Seiten der deutschen Gynäkologie, so habe ich nur einen plausiblen Grund dafür finden können: den gewaltigen Aufschwung, welcher in der chirurgischen Behandlung der gynäkologischen Leiden bei uns in den letzten beiden Decennien stattgefunden hat. $Z$ weifelsohne ist die Ausbildung der operativen Gynäkologie zu einer Höhe gelangt, wie man sie vor 20 Jahren kaum geahit, und $z$ weifelsohne hat sich die deutsche Gynäkologie ausserordentlich grosse Verdienste auf diesem Gebiete errungen.

Aber die einseitige Ausbildung der operativen Therapie, wie sie der deutschen Gynäkologie der letzten Decennien eigenthümlich ist hat auch meiner Ueberzeugung nach zu schädlichen Auswüchsen geführt und entschiedene Nachtheile für die Entwickelung der Gynäkologie mit sich gebracht. Anstatt auch andere Heilmethoden auszubilden, macht sich ein Streben geltend, alle Leiden auf operativem Wege zu lieilen.

Auf welchem Gebiete der Medicin ist es sonst üblich, wegen des Katarrhs einer Schleimhaut die ganze Schleimhaut zu exstirpiren, wie es noch vielfach beim Cervixkatarrh mit der Amputation der Vaginalportion geschieht! Manches Ovarium könnte noch am Ligamentum ovarii seiner Besitzerin sitzen, wenn der Operateur ge- 
lernt hätte, auch andere Methoden als die Exstirpation, z. B. die Massage oder die Elektrotherapie, zur Heilung der Ovarialschmerzen anzuwenden. $\mathrm{Ja}$ es führt diese einseitige operative Richtung sogar zu einer gewissen Vernachlässigung der Diagnostik, indem man sich auf die Laparotomie als Probeincision vielleicht manchmal allzusehr verliess. Mit Recht hat v. Bergmann diese Handlungsweise der Chirurgie in seiner Rede auf der Berliner Naturforscherversammlung mit dem Wort von ,der anticipirten Section" gegeisselt.

So ist es gekommen, dass andere Nationen gynäkologische Heilmethoden ausbildeten, welche bei uns gänzlich vernachlässigt wurden. So wurde die Massage, welche in anderen Zweigen der Medicin schon seit langer Zeit glänzende Erfolge errang, uns von Schweden aus eingeführt. So zeichneten sich die Amerikaner auf dem Gebiete der inneren Therapie der Frauenkrankheiten aus und brachten uns neue Heilmittel, z. B. Hydrastis. So ist die Einführung der Elektrotherapie in die Gynäkologie das Verdienst der Franzosen.

Es ist nicht meine Absicht, das gesamınte Gebiet der gynăkologischen Elektrotherapie hier in Betrachtung zu ziehen. Ich wollte Ihre Aufmerksamkeit nur auf einige Anwendungsweisen des faradischen Stromes in der Behandlung der Frauenkrankheiten richten.

In der Gynäkologie wurde der faradische oder inducirte Strom zuerst von Tripier ${ }^{1}$ ) angewendet. Tripier suchte damals die Ursache für alle Erkrankungen des Uterus in der mangelhaften Thätigkeit der Muskulatur desselben; er kannte ja die Infection als Ursache noch nicht, er betrachtete die durch Erschlaffung der Muskulatur erzeugte Congestion und Stauung als die Quelle aller Leideu und suchte durch Erregung der Muskulatur, durch Auregung der Circulation diese Leiden zu heilen. So glaubte er, indem er von falschen Voraussetzungen ausging, im faradischen Strom für die meisten Erkrankungen des Uterus ein Heilmittel gefunden zu haben. Uns kommen jetzt, nachdem wir in der Kenntniss der Aetiologie der Krankheiten so grosse Fortschritte gemacht haben, seine Anschauungen und seine aus diesen abgeleiteten therapeutischen Maassnahmen zum Theil absurd vor. Dennoch ist ihm das Verdienst nicht abzusprechen, auf die Wirksamkeit des faradischen Stromes auf die Uterusmuskulatur aufmerksam gemacht und den Anstoss zu einer weiteren Ausbildung der Elektrotherapie in der Gynäkologie gegeben zu haben. Sein Schüler ist A postoli, und ebenso wie dieser in ausgedehnter Weise den galvanischen Strom zur Behandlung der Frauenkrankheiten verwendet, hat auch er den faradischen Strom der gynäkologischen Therapie dienstbar zu machen gesucht und über seine Wirkungen mehrere Arbeiten veröffentlicht. ${ }^{2}$ )

Tripier wandte zur Faradisation des Uterus nur die unipolare Methode an, la méthode utéro-sus-pubienne, bei welcher der eine sondenförmige $\mathrm{Pol}$ in den Uterus eingeführt, die andere Elektrode auf den Unterleib gelegt wird. Apostoli führte eine neue Methode der Uterusfaradisation ein, die bipolare, indem er beide Pole in einer sondenförmigen Elektrode vereinigte. A postoli machte ausserdem darauf aufmerksam, dass, je nach der Beschaffenheit der secundären Rolle des faradischen Stromes, die Wirkung desselben eine ganz verschiedene ist, und zwar ist die Wirkung abhängig von der Dicke and Länge (Zahl der Umwindungen) des die secundäre Rolle umspinnenden Drahtes.

So viel ich weiss, haben die deutschen Elektrotherapeuten in Frankreich ist es schon länger bekannt - bis jetzt ihre Aufmerksamkeit auf diese Verschiedenheit der faradischen Ströme noch nicht gerichtet. Wenigstens habe ich in den Handbüchern der Elektrotherapie vergebens danach geforscht. Erb allein mach ${ }^{3}$ ) eine darauf bezügliche Bemerkung. Er sagt: „Es will mir immer scheinen, als seien secundäre Spiralen von sehr dünnem Draht weniger geeignet, weil sie entschieden schmerzhaftere Ströıne liefern."

Der Unterschied in der Beschaffenheit der faradischen Ströme, welche von verschiedenen secundären Rollen erzeugt werden, ist folgender.

1) Hyperplasie conjonctive des organes contractiles. De l'emploi de la faradisation dans le traitement des engorgements et déviations de l'uterus et de l'hypertrophie prostatique. Comptes rendus de l'Académie des sciences, Août 1859 .

3) Sur une nouvelle application de l'électricité après les accouchements. Communication faite à l'académie de médecine de Paris, 19. Avril 1881, par le Dr. G. Apostoli. - Sur un nouveau traitement de métrite chronique p. 4. - Sur un nouveau traitement électrique de la douleur ovarienne chez les hystériques. Bulletin général de thérapeutique, 15. Juin 1883 et Archives de tocologie 1885 . - Sur un nouveau traitement électrique des périmétrites. Lecture faite au Congrès médical international de Copenhague. Aoút 1884. Section d'obstétr. et de gynéc. Comptes rendus p. 141. Sur la faradisation double ou bipolaire. Communication faite à la société de médecine de Paris. Le 28. Arril 1883. L'union médicale du 28. Octobre et du 1. Novembre 1884. - On some new applications of the induced or faradic current in Gynaecologie. British Medical Journal 1888, Vol. I, p. 63,16 . Januar $1888 .{ }^{3}$ ) 1. c. p. 28 .
Nimmt man eine Rolle, welche mit dickem und nicht $z u$ langem Draht umsponnen ist, - ich habe gewöhnlich zu meinen Untersuchungen eine Rolle, wie ich sie Ihnen hier zeige, mit $400 \mathrm{Um}$ windungen eines $1 \mathrm{~mm}$ dicken Drahtes genommen -, so erhält man einen Strom, welcher vor allem erregend auf die Uterusmuskulatur wirkt; er ruft Uteruscontractionen hervor, regt die Circulation dadurch an, und wurde schon von Tripier zu diesem Zwecke angewendet.

Nimmt man aber eine secundäre Rolle mit dünnem und sehr langem Draht, - ich habe gewöhnlich eine Rolle mit 2500-3000 Windungen eines $0,25 \mathrm{~mm}$ dicken Drahtes gebraucht - , so erhält man einen Strom von höherer Spannung. A postoli nemnt ihn le courant de tension. Dieser Strom wirkt weniger auf die Muskulatur, als auf die Sensibilität. Er dient hauptsächlich dazu, den Schmerz zu stillen.

Man kann sich bei ein und derselben Patientin leicht von der Verschiedenheit der Wirkung dieser Ströme überzeugen. Faradisirt man den Uterus, sei es, dass man einen Pol einführt oder die bipolare Sonde benutzt, mit einem von der zuerst beschriebenen secundären Rolle erzeugten Strom, so wird man, wenn man die secundäre der primären Rolle nähert, gar bald heftige, wehenartige Schmerzen hervorrufen, welche durch die Uteruscontractionen bedingt sind. Zuweilen fühlt man den Uterus sich um die sondenförmige Elektrode zusammenziehen.

Applicirt man aber den von der mit dünnem und langem Draht umsponnenen Rolle hervorgerufenen Strom, so wird man, wenn man die secundäre Rolle langsam und allmählich der primären nähert, sehr wenig oder gar keine Schmerzen hervorrufen, abgesehen von den ziehenden Schmerzen, welche bei manchen Patientinnen die Einführung der Sonde an und für sich macht. Selten, und dann nur kurze Zeit dauernd, treten Contractionen auf. Dagegen wirkt der Strom ausserordentlich schmerzstillend und beruhigend bei Entzündungsprocessen in der Umgebung des Uterus.

Was nun die Methoden der Anwendung des faradischen Stromes auf den Uterus und die ihn nmgebenden Organe anbetrifft, so kann man von der Vagina und vom Uterus aus auf sie einwirken. Man kann den einen $\mathrm{Pol}_{0}$ in die Scheide, und zwar als kugelförnige, oder in den Uterus, als sondenförmige Elektrode, den anderen auf die Bauchdecken in Gestalt einer möglichst grossen Platte (400 qcm babe ich gewöhnlich genommen, um möglichst starke Ströme anwenden zu können) appliciren. $\mathrm{Da}$, wo ich schmerzstillend wirken wollte, habe ich gewöhnlich die Anode des Oeffnungsstromes in die Genitalien eingeführt.

Die bipolare vaginale Methode, wie sie Apostoli für gewisse Zwecke empfiehlt, habe ich nicht prüfen können, da es mir, oder vielmehr Herrn Hirs ch man ${ }^{1}$ ) dem Elektrotechniker, bis jetzt noch nicht gelungen, eine geeignete bipolare vaginale Elektrode $\mathrm{zu}$ construiren. Alle die vaginalen Elektroden, welche wir versuchten, - Herr Hirschmann hatte auch nach den Apostoli'schen Zeichnungen Elektroden construirt - , waren, sobald sie von dem Vaginalsecret bedeckt waren, nicht mehr isolirt. Die Stromkette schliesst sich dann, ohne dass der Strom den Körper der Patientinnen passirt, wie man nach Herausnahme der Elektrode mittels des constanten Stromes am Galvanometer sehen kann.

Dagegen ist es nach mehreren vergeblichen Versuchen auf meine Anregung hin Herrn Hirschmann gelungen, eine sehr brauchbare intrauterine bipolare Sonde zu construiren - die Apostoli'sche kenne ich nicht. In dieser Sonde bleiben die Pole isolirt, auch wenn sie vom Uterussecret bedeckt ist, so dass kräftige Stromschleifen von der Uterushöhle aus das weibliche Becken treffen.

Natürlich muss man auch diese Sonde $a b$ und zu mittels des constanten Stromes untersuchen, um zu sehen, ob die Isolation noch vollkommen ist.

Mit dieser Sonde konnte ich die bipolare intrauterine Faradisation einer genauen Untersuchung unterziehen, und ich kann die Vortheile, welche Apostoli dieser Methode nachrühmt, nur bestätigen. Sie ist entschieden einfacher auszuführen, da man Niemandes Hülfe bedarf, um die äussere Elektrode zu halten, schmerzloser, da die äussere Haut bei ihr nicht afficirt wird, und anscheinend wirksamer in ihren therapeutischen Erfolgen.

Betrachten wir nun die Anwendung des faradischen Stromes zu therapeutischen $Z_{w e c k e n}$ in der Gynäkologie, so habe ich den Uteruscontractionen hervorrufenden Strom bisher nur wenig angewendet und geprüft, um über seinen therapeutischen Werth ein definitives Urtheil fällen zu können. Man muss, um Uteruscontractionen zu erzielen, intrauterin faradisiren, sei es nun nach der unipolaren oder bipolaren Methode.

Von der Vagina aus oder beim Ansetzen eines Poles an die Vaginalportion erhält man keine, wenigstens nachweisbaren Uteruscontractionen. Dagegen erzielt man bei der intrauterinen Anwen- 
dung des inducirten Stromes sehr bald Contractionen. Leider sind wir nicht in der Lage, wenigstens bis jetzt, die auftretenden Contractionen direktzu messen, sie graphisch darzustellen. Selbst eine Erhärtung des Uterus direkt durch die bimanuelle Untersuchung nachzuweisen, wird nur in den wenigsten Fällen gelingen. Zuweilen hat man wohl das Gefühl, als ob sich der Uterus um den sondenförmigen Pol zusammenzieht. Aber im allgemeinen ist man bei der Beurtheilung der Wirkung des Stromes auf die subjectiven Symptome, auf die Angaben der Patientinnen, welche über ziehende, wehenartige Schmerzen klagen, die im Kreuz, im Leibe auftreten bis in die Oberschenkel hinabziehen, angewiesen.

Apostoli') empfiehlt die Anwendung des faradischen Stromes sehr zur Behandlung der Subinvolutionen; auch $\mathrm{Bumm} \mathrm{m}^{2}$ ) hat bei der Behandlung dieses Leidens nicht ohne Erfolg den Strom gebraucht. Ich besitze keine Erfahrungen hierüber. Doch bin ich der Ueberzeugung, dass die Anwendung nützlich sein wird, da der faradische Strom jedenfalls sicherer als Secale oder heisse Einspritzungen energische Uteruscontractionen herbeizuführen im Stande ist. Auch für die Uterusatrophie, sowie für die Amenorrhoe lassen sich von der die Blutcirculation anregenden Wirkung des Stromes noch Erfolge erhoffen. Doch sind meine Versuche hierüber noch nicht abgeschlossen.

Dagegen habe ich den auf die Sensibilität wirkenden Strom, welcher von einer secundären Rolle mit zahlreichen Umwindungen eines dünnen Drahtes erzeugt wird, einer Prüfung unterzogen und bin $\mathrm{zu}$ Resultaten gelangt, welche ich hier mitzutheilen mir erlauben werde.

Es wirkt dieser Strom im hohen Grade, und zwar dauernd schmerzstillend, und da ja bei sehr vielen Frauenkrankheiten der Schmerz das Symptom ist, welches die Kranken zum Arzt führt, so ist der faradische Strom, welcher dieses Symptom in den meisten Fällen mit absoluter Sicherheit zu bekämpfen im Stande ist, ein Mittel, welches werth ist, in ausgedehnter Weise in der gynäkologischen Therapie angewendet zu werden.

Die schmerzstillende Wirkung elektrischer Ströme, speciell des faradischen, ist den Elektrotherapeuten längst bekannt. So theilt Moritz Meier in seinem Handbuch der Elektrotherapie 1883, p. 100, einen Fall von Coccygodynie mit, welcher 12 Jahre bestand und in einer Sitzung mit dem faradischen Strome geheilt wurde. Vor allem ist es der von den Ovarien ausgehende Schmerz, sei es nun, dass er entzündlicher oder nervöser Natur ist, für welchen der iuducirte Strom in den meisten Fällen ein absolut sicheres Heilmittel ist.

Dass die Zahl der Kranken, welche an Ovarialschmerzen leiden, nicht gering ist, dass unsere Therapie diesen Beschwerden gegenüber ziemlich machtlos war, brauche ich hier kaum hervorzuheben.

Man findet in solchen Fällen bei der genauen bimanuellen Untersuchung das Ovarium druckempfindlich, oft so sehr, dass eine einzige Untersuchung tagelang dauernde heftige Beschwerden hervorruft; gewöhnlich nur auf der einen Seite erkrankt, manchmal auch auf beiden, zuweilen vergrössert bis zu Hühnereigrösse, manchmal ohne erhebliche Vergrősserung, bald weicher, bald hart sich anfühlend. Zuweilen ist das Ovarium dislocirt, in den Douglas descendirt. Kurz, es handelt sich gewöhnlich dabei um jenen Symptomencomplex, welchen die Autoren als chronische Oophoritis bezeichnen und als eine locale Peritonitis auffassen. In der Mehrzahl der Fälle finden wir auch Residuen der Perimetritis in der Umgebnng der Ovarien; dieselben sind von Schwarten bedeckt,durch Adhäsionen fixirt (Perioophoritis). Häufig sind die Tuben miterkrankt, mit den Eierstöcken verwachsen; es findet sich dabei Perimetritis posterior etc.

Die Patientinnen haben oft ganz unerträgliche Beschwerden von ihrem Leiden; Schmerzen, welche im Becken sitzen, nach den Beinen, nach den Hypochondrien ausstrahlen, ja die ganze Körperhälfte ergreifen können. Wie viele von diesen Fällen als rein entzündliche, wie viele als Neuralgieen des Ovariums aufzufassen sind, ist schwer zu sagen. Wissen wir doch über die pathologische Anatomie der chronischen Oophoritis noch wenig, und fassen manche Autoren, wie z. B. Band $1^{3}$ ), sogar die Ovarie, jenen bei Hysterischen am eingehendsten von Charcot beschriebenen Symptomencomplex, als eine locale Peritonitis auf.

Diesen so hăufig sich findenden Ovarialschmerz kann man mit grosser Sicherheit mittels des faradischen Stromes heilen. Schon wenn man einen Pol, z. B. gegen ein descendirtes und fixirtes Ovarium setzt, gelingt es, wenn man die Sitzung nur lang genug ausdehnt, das vorher ungeheuer empfindliche Ovarium, welches der

1) Sur un nouveau traitement de la métrite chronique. Paris 1887, Octave Doin.

2) Untersuchungen über die elektrische Reizbarkeit des Uterus bei Schwangeren, Kreissenden und Gebärenden. Archiv für Gynäkologie Band XXIV, p. 64 .

3) Billroth-Lücke, Handbuch der Frauenkrankheiten Theil II, p. 927.
Patientin beim Gehen, bei der Defäcation, bei der Cohabitation heftige Schmerzen verursachte, entweder ganz unempfindlich oder jedenfalls sehr viel weniger empfindlich zu machen. Noch besser kann man dieses mittels der bipolaren intrauterinen Faradisation erreichen. Vom Uterus aus gelingt es am besten auf die Ovarien mit dem inducirten Strom zu wirken. Von hier aus können, so stelle ich mir die Sache vor, jedenfalls am besten starke Stromschleifen zu den Ovarien dringen. Warum der faradische Strom gerade auf die Nerven des Ovariums so stark einwirkt, ist schwer zu sagen. Wissen wir doch überhaupt über die Wirkung elektrischer Ströme auf die Träger der Schmerzempfindungen, auf die sensiblen Nerven, noch herzlich wenig.

Die vaginale Faradisation wird nur da angewendet, wo sich eine Sondirung der Uterushöhle überhaupt verbietet, also bei acuten und subacuten Entzündungen und bei Gravidität, sonst aber ist die uterine Faradisation, und zwar am besten die bipolare Methode zu gebrauchen.

Naclıdem man die in 5\% iger Carbollösung desinflcirte Sonde in den Uterus eingeführt - ich habe sie aus antiseptischen Gründen gewöhnlich im Milchglasspeculum eingeführt - und die Leitungsschnüre befestigt hat, lässt man den Strom gauz allmählich anschwellen, indem man die secundäre Rolle langsam von Millimeter zu Millimeter der primären Rolle nähert und dabei das Gesicht der Kranken genau beobachtet. Deun es ist überflüssig und schädlich, ihr Schmerzen zu machen. Auf diese Weise kann man allmählich eine ganz ausserordentlich grosse Stromstärke erreichen, ohne dass die Patientinnen irgend welche Beschwerden haben, eine so grosse Stromstärke, wie wir sie auf der äusseren Haut gar nicht vertragen können. Manche Kranken sind so wenig empfindlich bei dieser Anwendungsweise des Stromes, dass man während der Sitzung glaubt, der Apparat functionire nicht. Andere sind empfindlicher. Bei diesen muss man den Strom sehr vorsichtig anschwellen lassen.

Um sichere Erfolge zu erzielen, muss man möglichst starke Ströme anwenden und die Sitzungen möglichst lange ausdehnen. Besonders die erste Sitzung muss so lange ausgedehnt werden, bis eine wirkliche Besserung oder ein gänzliches Verschwinden der Schmerzen erreicht wird. Das kann unter Umständen schon nach 5 Minuten geschehen, manchmal aber auch erst nach 10-20 Minuten.

Die folgenden Sitzungen brauchen, wenn die erste ordentlich gewirkt hat, nicht so lange zu dauern, durchschnittlich 7-10 Minuten wird man aber auch die Elektricität wirken lassen müssen.

Was die Zahl der Sitzungen anbetrifft, welche nöthig sind, um ein dauerndes Resultat zu erreichen, so ist dieselbe ebenfalls sehr verschieden. Beí eíner Patientín z. B., welche seit 6 Monaten an Beschwerden litt, die durch eine doppelseitige Oophoritis und Perioophoritis bedingt waren, genügten 4 Sitzungen, um sie zu heilen, bei einer anderen, welche an einer linksseitigen Oophoritis und Perioophoritis litt, einem sehr hartnäckigen Falle, den ich schon Monate lang vergebens massirt hatte, waren 35 Sitzungen nöthig, um sie anscheinend dauernd von ihren Beschwerden zu befreien.

M. H.! Ich will Sie hier nicht mit Krankengeschichten langweilen. Ich habe im ganzen 25 Fälle von chronischer Oophoritis mit dem faradischen Strom behandelt. Von diesen waren 8 Fälle mit Retroflexio complicirt, 7 puerperaleu, 5 zweifelsohne und 3 wahrscheinlich gonorrhoischen Ursprungs. Bei zwei Fällen war die Ursache nicht sicher zu eruiren.

Die Fälle waren mehr oder weniger mit Entzündungen der Tuben, der Ligamenta sacro-uterina, der Ligamenta lata complicirt, wie das ja immer der Fall zu sein pflegt. Doch waren in allen diesen Fällen sowohl die subjectiven Beschwerden, als auch der objective Befund derart, dass man annehmen musste, dass das Ovarium der Hauptsitz der Erkrankung war.

Nur drei Fälle waren, soweit man das durch die bimanuelle Untersuchung nachweisen konnte, nicht mit Entzündungen anderer Stellen in der Umgebung des Uterus complicirt. Diese Fälle waren alle drei bald nach dem Puerperium entstanden, ohne dass eine Perimetritis im Wochenbett beobachtet war. In 14 Fällen handelte es sich um einseitige, in $\mathbf{1 1}$ um doppelseitige Erkrankungen.

In drei Fällen war der Uterus retroflectirt und fixirt, die Ovarien descendirt und fixirt.

Von diesen Fällen sind 21 dauernd von ihren Beschwerden befreit, $d$. h. so lange, wie ich sie bis jetzt beobachtet habe, 3 bis 4 Monate, - ich habe mir die Kranken, soweit ich ihrer noch habhaft werden konnte, vor dem Abschluss dieses Vortrages in die Poliklinik bestellt und sie untersucht -, die übrigen 4 Fälle wurden wesentlich gebessert, zwei davon sind noch in Behandlung.

Anatomisch trat eiue nachweisbare Veränderung nicht ein, ein fixirtes Ovarium blieb fixirt, ein vergrōssertes fast immer vergrössert, aber die Druckempfindlichkeit verschwand entweder ganz oder blieb sehr gering, die subjectiven Beschwerden aber waren ganz beseitigt. 
Darunter waren sehr schwere Fälle; mehrere von ihnen hatte ich Monate lang vorher vergeblich mit Massage behandelt; bei \&wei Fällen, welche jeder anderen Therapie Trotz geboten hatten, war ich schon entschlossen gewesen, die Exstirpation der Ovarien vorzunehmen, bevor ich die bipolare intrauterine Faradisation anwendete. Einzelne Kranke konnten nicht mehr gehen und waren gänzlich arbeitsunfähig. Bei einem Fall von Retroflexio, welcher wegen der Complication mit Oophoritis duplex gar kein Pessar vertragen konnte, gelang es, nachdem die Ovarien durch die bipolare intrauterine Faradisation unempindlich gemacht waren, ein Pessar einzulegen, welches die Kranke dann von ihren Beschwerden befreite.

Auch intrauterine Eingriffe, z. B. die Aetzung der Uterusschleimhaut mit Chlorzink, welche ich vorher wegen der Empfindlichkeit und Schmerzhaftigkeit der Ovarien nicht vorzunehmen wagte, wurden jetzt sehr gut vertragen.

In einzelnen Fällen musste ich, um die bipolare Sonde einführen zu können, - dieselbe ist nicht gan -, den Uterus vorher erweitern. Das kann man am schonendsten mittels der Kathode des constanten Stromes, indem man allmählich immer dickere Aluminiumsonden als activen Pol einführt. Dann bleibt der Uteruscanal dauernd genügend weit, die Dilatation bleibt bestehen in Gegensat\% zu anderen Erweiterungsmethoden; dazu genügt manchmal nur eine, manchmal $3-4$ Sitzungen.

Ich halte den faradischen Strom, in geeigneter Weise angewendet, für ein specifisches Mittel gegen alle vom Ovarium ausgehenden Schmerzen, sei es nun, dass sie entzündlicher oder mehr neuralgischer Natur sind, und bin der Ueberzeugung, dass manche Castration gespart werden kann, wenn man diese Behandlungsmethode zur Beseitigung der Ovarialschmerzen anwendet.

Nicht ganı so günstig, aber doch in vielen Fällen von vortrefflichen Erfolgen begleitet, war die Anwendung des faradischen Stromes bei den übrigen chronischen Entzündungen in der Umgebung des Uterus, bei den Residuen der Peri- und Parametritis.

Auch hier zeigten sich die schmerzstillenden, beruhigenden, ich möchte fast sagen antiphlogistisch wirkenden Eigenschaften der inducirten Elektricität. So habe ich z. B. an einem Fall von acuter Pelveoperitonitis, welcher ein grosses Exsudat bekam, mich von der schmerzstillenden Wirkung überzeugen können. Bei der Patientin, welche mit Fieber und starken Unterleibsschmerzen erkrankt war, war beim Touchiren die Douglas'sche Tasche, das gan gewölbe ungeheuer empfindlich. Ich liess 20 Minuten den faradischen Strom, die Anode des Oeffnungsstromes in der Vagina, den anderen $\mathrm{Pol}$ auf die Bauchdecken, und zwar so stark als möglich wirken. Hinterher war die Empfindlichkeit fast ganı verschwunden, die Kranke fühlte sich sehr erleichtert.

Hauptsächlich aber habe ich die Residuen der acuten Entzündungen behandelt. Bei diesen Erkrankungen ist es ja auch der Schmerz, welcher die Kranken zum Arzt führt; der beständige Druck im Becken nach unten, das Brennen im Leibe, die mannichfachen Reflexneurosen, das Ausstrahlen der Sclimerzen nach dem Kreuz nach den Beinen hin etc. Auf alle diese Symptome wirkt der inducirte Strom sehr günstig, die Schmerzen lindernd, vertreibend, und auch eine Rückwirkung auf das gesammte Nervensystem ist bei den Patientinnen, bei welchen diese Beschwerden so häufig mit mannichfachen neurasthenischen Symptomen verbunden sind, in manchen Fällen nicht zu verkennen. Allerdings gelingt es selten, so schnell wie beim Ovarialschmerze die Beschwerden zu vertreiben. Während dazu oft 4-5 Sitzungen genügen, sind in den meisten Fällen von periuteriner Entzündung zahlreiche Sitzungen nöthig, um nur eine Besserung des Zustandes zu erzielen.

In einzelnen Fällen erreicht man allerdings ganz überraschende Resultate.

Bei einer Patientin z. B., Frau A. H., welche eingestandenermaassen gonorrhoisch und syphilitisch inficirt war, waren in einer gynäkologischen Klinik im Februar 1886 wegen starker Unterleibsschmerzen die linken Anhänge exstirpirt. Es fand sich, wie mir mitzutheilen die Assistenten der Klinik die Güte hatten, eine linksseitige Salpingitis catarrhalis mit beginnendem Hydrops, Oophoritis chronica sinistra, linksseitige Parovarialcyste. Danach dreimonatliches Wohlbefinden. Am 10. Mai wieder Beschwerden rechts. Rechtes Ovarium vergrössert. Tube verdickt. 14. September 1886 faustgrosser Tumor rechts. 20. September 1886 Laparotomie. Entfernung der rechten Anhänge. Hydrosalpinx dextra. Hydrops follicul. ovarii. Cyste parvoarii dextri. Patientin fühlte sich nur ganz kurze Zeit wohl danach. Es traten wieder heftige Beschwerden ein, so dass sie die Hülfe eines anderen Gynäkologen in Anspruch nahm. Es wurde von ihm, wie er mir mitzutheilen die Güte hatte, wegen der überaus hochgradigen Beschwerden der Kranken im November 1887 der Uterus exstirpirt. Ich sah die Kranke dann im März 1889. Sie war über ein Jahr nach der letzten Operation ohne Beschwerden. Jet/t litt sie wieder seit 8 Wochen an ganz unerträ́glichen Schmerzen im Leibe, welche bis in die Beine ausstrahlten. Bei der Untersuchung fand ich im Scheidengewölbe eine quer verlaufende Narbe, welche ungeheuer empfindlich war. Das ganze Beckenperitoneum war bei der bimanuellen Untersuchung äusserst empfindlich. Die Genitalorgane fehlten. Exsudatmassen, Addhäsionen etc. waren nicht zu fühlen. Ich beobachtete die Frau bis zum 21. Märı Ihre Beschwerden waren ausserordentlich gross. Am 21. März applicirte ich zum ersten Mal den faradischen Strom (kugelförmige Elektrode in die Vagina) 15 Minuten lang. Die Kranke fühlte sich nach der Sitzung sehr erleichtert.

Vom 22. bis 27. März noch 3 Sitzungen von 10 bis 12 Minuten Dauer. Danach waren die Schmerzen gan\% verschwunden, die Narbe, das Becken gan ich sie zuletzt sah, war sie noch gan\% ohne Beschwerden.

Weder die Exstirpation der Anhänge, noch die Exstirpation des Uterus war im Stande gewesen, die Kranke dauernd zu heilen. Es steht dieser Fall in direktem Gegensatz zu den Behauptungen Laws on Tait's über die Wirkung der Entfernung der Uterusanhänge.

Bei einer anderen Patientin, einer 21 Jahre alten Frau, welche gonorrhoisch inficirt war, war wegen ihrer Perimetritis von anderer Seite einer jener kleinen Ovarialtumoren exstirpirt. Doch hatte sie ihre Beschwerden behalten. Sie war dann eine Zeit lang in einer anderen Klinik mit dem constanten Strom behandelt, ohne nennenswerthen Erfolg. Sie kam Anfangs Februar dieses Jahres in meine Behandlung. Sie hatte eine nicht unbeträchtliche Bauchnarbenhernie. Uterus nach links. Linke Anhänge sehr empfindlich. Rechte Anhänge fehlen. Rechts oben in der Gegend der Linea innominata weiche gurrende Resistenzen, starker eitriger Fluor. Sie hatte sehr starke Beschwerden, starke Dysmenorrhoe, Brennen im Unterleib. Das Einführen der bipolaren Sonde war schwierig, da die Patientin sehr empfindlich, der ganze Uteruscanal, besonders das Orificium internum sehr eng war.

Ich erweiterte ihr in fünf Sitzungen zuerst den Uterus mittels der Kathode des galvanischen Stromes. Danach verschwand die Dysmenorrhoe. Dann wandte ich die bipolare intrauterine Faradisation an. In 10 Sitzungen wurde die Kranke von ihren Beschwerden befreit, die Beckenorgane, die Resistenzen im Becken unempfindlich, der Fluor blieb bestehen. Vom 29. Märı bis 28. Juni blieb sie ohne Beschwerden. Am 28. Juni hatte sie wieder seit 8 Tagen etwas Beschwerden. Wiederum waren 10 Sitzungen nöthig, um sie von ihren Beschwerden zu befreien. Seit Ende Juli ist sie schmerzfrei, sodass ich sie dann von ihrem Fluor durch intrauterine Chlorzinkätzungen befreien konnte.

Hier hatte der faradische Strom, nachdem der constante nicht geholfen, sehr günstig gewirkt. Dass bei derartigen Fällen auch nach der Anwendung der Elektricität Reridive auftreten, ist bei dem chronischen Charakter dieser Erkrankungen kein Wunder.

Ich habe bisher 21 Fälle von Residuen der Peri- und Parametritis mit dem faradischen Strom behandelt, und zwar theils mit der vaginalen unipolaren, theils mit der bipolaren intrauterinen Faradisation. Von diesen 21 Fällen sind 10 dauernd, d. h. 4-5 Monate, so lange ich sie beobachtete - - von ihren Beschwerden befreit. 4 Fälle wurden bedeutend, 4 Fälle etwas gebessert. In drei Fällen sah ich gar keinen Erfolg.

Die meisten dieser Fälle waren mit Metritis, Endometritis complicirt. In mehreren bestand Retroflexio fixata.

Zwei Mal wandte ich die Faradisation in der Gravidität an, das eine Mal im zweiten Monat der Schwangerschaft wegen Perimetritis posterior und Perioophoritis, das andere Mal im 3. bis 4. Monat wegen grosser Empfindlichkeit der Iunenfläche des Kreuzund Steissbeines, beide Male mit gutem Erfolge hinsichtlich der Beschwerden und ohne Schaden für die Schwangerschaft. In sehr vielen Fällen waren alle anderen Mittel vergebens angewendet, die Elektricität das ultimum refugium.

Nochmals betone ich, dass in keinem Falle die anatomischen Verhältnisse durch den faradischen Strom nachweisbar verändert wurden, ein verdicktes Ligament blieb verdickt, ein fixirter Uterus fixirt, aber die Druckempfindlichkeit und die subjectiven Beschwerden verschwanden. Es sind gegen die Residuen der Parametritis und Perimetritis Versuche von 0 rthmann ${ }^{1}$ ) mit dem galvanischen Strom gemacht. Ich glaube, dass meine Resultate, besonders in Be\%ug auf die chronische Oophoritis, besser sind. So weit meine Erfahrungen reichen, ist der galvanische Strom zur Förderung der Resorption circumscripter, grösserer Exsudate besser geeignet. Gegen die Residuen der Peri- und Parametritis aber ist der faradische Strom das geeignetste Mittel, um die Beschwerden zu lindern oder $\% u$ beseitigen.

Noch eins möchte ich hervorheben. In allen meinen Fällen habe ich den faradischen Strom allein, ohne die übrigen Hülfsmittel der gynäkologischen Therapie angewendet, um einwandsfreie Beob-

1) Berliner klin. Wochenschrift 1889, No. 21. 
achtungen zu erhalten. Ich bin der Ueberzeugung, dass die Verbindung der Anwendung des faradischen Stromes mit der Hydrotherapie, mit der Massage noch bessere Erfolge geben wird. So wird es sich empfehlen, Badecuren durch die Elektricität zu unterstützen, Narbenstränge, Verwachsungen u. s. w. unempfindlich zu machen, bevor man sie mittels der Massage auszieht und beseitigt. Der Therapie dieser chronischen Leiden eröffnen sich so neue and Erfolge versprechende Aufgaben. 